\title{
ON ARNOLD'S FORMULA FOR THE DIMENSION OF A POLYNOMIAL RING
}

\author{
PAUL EAKIN ${ }^{1}$
}

ABSTRACT. If $R$ is a commutative integral domain with quotient field $K$ and $x_{1}, \ldots, x_{n}$ are indeterminates, then there exist $\theta_{1}, \ldots, \theta_{n}$ in $K$ such that $\operatorname{dim} R\left[x_{1}, \ldots, x_{n}\right]=n+\operatorname{dim} R\left[\theta_{1}, \ldots, \theta_{n}\right]$.

If $R$ is a commutative ring, the Krull dimension of $R$ is the maximum of the lengths of all chains of prime ideals in $R$. If $R=\mathcal{C}[V]$ is the coordinate ring of an affine variety $V$ over the complex numbers, then increasing chains of primes in $R$ correspond to decreasing chains of irreducible subvarieties. In this "geometric case" the Krull dimension corresponds to our intuitive notion of (complex) topological dimension. Moreover, since $R[X]$ corresponds to $V \times \circlearrowright$ (the product of $V$ and an affine line), intuition would lead us to suspect

$$
\operatorname{dim} R[X]=\operatorname{dim} R+1 .
$$

In [7], W. Krull established (*) for any noetherian ring. Seidenberg [9], [10] investigated the validity of $(*)$ for arbitrary commutative rings and observed that it does not hold in general. He observes that one always has

$$
\operatorname{dim} R+1 \leqslant \operatorname{dim} R[X] \leqslant 2 \operatorname{dim} R+1,
$$

and he provides examples to show that within these bounds anything can happen.

Jaffard [6] made an extensive study of the dimension theory in polynomial rings. He introduced the notion of valuative dimension of a domain $R$. This is just the maximum of the ranks of the valuation overrings of $R$. Jaffard showed that when $(*)$ fails, the valuative dimension of $R$ must exceed the dimension of $R$. In addition, he studied the asymptotic behavior of the function $f(n)=\operatorname{dim} R\left[X_{1}, \ldots, X_{n}\right]$ and showed that if $R$ is a domain of finite valuative dimension, then for all suitably large $n$ one has $f(n+1)=f(n)+1$.

In [4] Gilmer and Bastida call the sequence $\{f(i)\}_{i=0}^{\infty}$ the dimension sequence of the ring $R$, and they investigate which sequences are dimension sequences of a certain class of rings. In [2] Arnold and Gilmer determine all sequences which are the dimension sequence of a commutative ring.

Both [2] and [4] depend upon a result of Arnold [1, Theorem 5, p. 323] which we refer to as Arnold's formula. We state the result as follows:

Received by the editors September 12, 1974 and, in revised form, November 21, 1974.

AMS (MOS) subject classifications (1970). Primary 13A15, 13B25, 13C15, 13G05.

Key words and phrases. Krull dimension, polynomial ring.

${ }^{1}$ Supported by the National Science Foundation. 
If $R$ is a commutative integral domain with quotient field $K$ and $X_{1}, \ldots, X_{n}$ are indeterminants over $R$, then there exist $\theta_{1}, \ldots, \theta_{n}$ in $K$ such that

$$
\operatorname{dim} R\left[X_{1}, \ldots, X_{n}\right]=n+\operatorname{dim} R\left[\theta_{1}, \ldots, \theta_{n}\right] .
$$

One always has that in $(* *)$ the left-hand side is greater than or equal to the right-hand side. Thus the interesting fact is that the maximum possible dimension of the rings of the form $R\left[\theta_{1}, \ldots, \theta_{n}\right]$ can always be realized. The proof of the formula in [1] is, however, incorrect and we know of no correct proof in the literature. ${ }^{2,3}$ Our purpose here is to provide an elementary proof.

In what follows, all rings are assumed to be commutative and to possess an identity. When we write "dim $R$ " we are referring to the Krull dimension of the ring $R$. By $R\left[X_{1}, \ldots, X_{n}\right]$ we denote the ring of polynomials in the independent variables $\left\{X_{1}, \ldots, X_{n}\right\}$ over the ring $R$. Finally, whenever we use the symbol " $<$ " it is meant to denote strict containment.

Our argument requires a few well-known facts which we list for the convenience of the reader.

(A) Let $k$ be a field and $R=k\left[x_{1}, \ldots, x_{n}\right]$ a finitely generated ring extension of $k$. If $P$ is a prime of $R$, then $\operatorname{dim} R=\operatorname{rank} P+\operatorname{trans} \operatorname{deg}_{k}(R / P)[8, p .46$, (14.6)].

The following is observed by Seidenberg [9] and is a consequence of (A) applied to the ring of polynomials in $n$ variables over a field.

(B) If $\left\{P_{i}\right\}_{i=0}^{l}$ is a chain of primes in $R\left[X_{1}, \ldots, X_{n}\right]$ all of which lie over the same prime of $R$, then $l \leqslant n$.

(C) If $V^{*}$ is a valuation ring of rank $n$ with quotient field $L$, and if $L$ is of transcendence degree $d$ over the field $K$, then rank $\left(V^{*} \cap K\right) \geqslant n-d[3, p .440$, Corollary 2].

A chain $\mathscr{D}=\left\{P_{i}\right\}_{i=0}^{m}$ of primes in a polynomial ring $R\left[X_{1}, \ldots, X_{n}\right]$ is called a special chain if, for each $P_{i} \in \mathscr{D}$, the ideal $\left(P_{i} \cap R\right)\left[X_{1}, \ldots, X_{n}\right]$ is a member of $\mathscr{D}$.

(D) JAFFARD'S SPECIAL CHAIN THEOREM. If $Q$ is a prime ideal of $R\left[X_{1}, \ldots, X_{n}\right]$ of finite rank $r(Q)$, then $r(Q)$ can be realized as the length of a special chain of primes in $R\left[X_{1}, \ldots, X_{n}\right]$ with terminal element $Q$. In particular, if $R$ is finite dimensional then $\operatorname{dim} R\left[X_{1}, \ldots, X_{n}\right]$ can be realized as the length of a special chain of primes of $R\left[X_{1}, \ldots, X_{n}\right]$.

This is the statement of Jaffard's theorem in [5]. The authors give an argument there which we feel is much easier than Jaffard's proof [6].

Proof of Arnold's formula. Suppose there were a counterexample, say $R\left[X_{1}, \ldots, X_{n}\right]$. Then we may assume that $n$ is minimal and that for this fixed $n, R$ has minimal dimension. We must have that both $n$ and $\operatorname{dim} R$ are greater than zero: if $\operatorname{dim} R=0, R$ is a field and by (A) we could simply take $\theta$ 's $=$ 0 . If $n=0$ we could again take all $\theta$ 's $=0$.

\footnotetext{
${ }^{2}$ I wish to thank Jon Johnson for bringing the error to my attention.

${ }^{3}$ Bastida and Gilmer point out the error in [4]. However their discussion of the mistake is itself erroneous. In [4] they claim to remove the doubt about this formula by independently proving Theorem 3 of [1] (which has the incorrect proof). However their argument ultimately rests on Theorem 5 of [1] whose proof (in [1]) is based on Theorem 3 of [1].
} 
Let $s=\operatorname{dim} R\left[X_{1}, \ldots, X_{n}\right]$. By (D) there is a special chain $0<P_{1}<\cdots$ $<P_{t}<\cdots<P_{s}$ in $R\left[X_{1}, \ldots, X_{n}\right]$. Let $t$ be minimal such that $P_{t} \cap R \neq 0$. Let $P_{t} \cap R=q$. By (A), $t-1 \leqslant n$ and $P_{t}=q\left[X_{1}, \ldots, X_{n}\right]$ by the minimality of $t$. Since our chain has maximal length, rank $q\left[X_{1}, \ldots, X_{n}\right]=t$. Let $S=R \backslash q$ and localize $R\left[X_{1}, \ldots, X_{n}\right]$ at $S$ to get $T=R_{q}\left[X_{1}, \ldots, X_{n}\right]$. Now for any local ring $R_{q}$, it is an easy consequence of (D) that $\operatorname{dim} R_{q}\left[X_{1}, \ldots, X_{n}\right]=n$ $+\operatorname{rank} q\left[X_{1}, \ldots, X_{n}\right]$ (or see [5]). Thus $\operatorname{dim} T=n+t$.

Let $\bar{T}=R_{q}\left[X_{1}, \ldots, X_{n}\right] / P_{t-1} R_{q}\left[X_{1}, \ldots, X_{n}\right]$. Then $\operatorname{dim} \bar{T}=n+1$ and there exists a chain of primes

$$
0<q \bar{T}<Q_{2}<\cdots<Q_{n+t}<\bar{T} .
$$

Since $P_{t-1} \cap R=0$ we may identify $R$ with its image in $\bar{T}$ and assume that $K$, the quotient field of $R$, is contained in that of $\bar{T}$. Let $V^{*}$ be a valuation overring or $\bar{T}$ which is centered on the chain $(* * *)[8$, p.37, (11.9)]. Let $V=V^{*} \cap K$.

Claim. rank $V \geqslant t$.

Proof of Claim. We first compute the transcendence degree of $\bar{T}$ over $R$. To do this we may localize $\bar{T}$ at $s^{\prime}=R \backslash 0$. By the permutability of residue class ring and quotient ring formation

$$
\begin{aligned}
\bar{T}_{s^{\prime}} & \cong\left(R_{q}\left[X_{1}, \ldots, X_{n}\right] / P_{t-1} R_{q}\left[X_{1}, \ldots, X_{n}\right]\right)_{s^{\prime}} \\
& \cong K\left[X_{1}, \ldots, X_{n}\right] / P_{t-1} K\left[X_{1}, \ldots, X_{n}\right] .
\end{aligned}
$$

Since rank $P_{t-1}=\operatorname{rank} P_{t-1}\left(K\left[X_{1}, \ldots, X_{n}\right]\right)=t-1$, we have $\operatorname{dim} \bar{T}_{s^{\prime}}=[n$ $-(t-1)]$ (by (A)). Thus by (A) the transcendence degree of $\bar{T}$ over $R$ is $[n-(t-1)]$. Therefore we have by (C) that rank $(V) \geqslant(n+1)-[n-(t$ $-1)]=t$. This establishes the Claim.

Since each prime of the chain (***) contains $q$, it follows that each prime of $V$ contains $q$. Thus each prime of $V$ meets $R_{q}$ at $q R_{q}$. Let $M_{1}<\cdots<M_{t}$ be a chain in $V$ such that $M_{j} \cap R_{q}=q R_{q}$. For each $j$ such that $1 \leqslant j \leqslant t-1$, choose $\theta_{j} \in M_{j+1} \backslash M_{j}$.

Consider the canonical homomorphism $V \stackrel{\sigma}{\rightarrow} V / M_{1}$. Under $\sigma, R_{q}\left[\theta_{1}, \ldots\right.$, $\left.\theta_{t-1}\right]$ maps into $V / M_{1}$. Denote the field $R_{q} / q R_{q}$ by $k$ and let $\sigma\left(\theta_{j}\right)=\bar{\theta}_{j}$. Then under $\sigma$ we have

$$
R / q\left[\bar{\theta}_{1}, \ldots, \bar{\theta}_{t-1}\right] \subset k\left[\bar{\theta}_{1}, \ldots, \bar{\theta}_{t-1}\right] \subset V / M_{1} .
$$

By our choice of the $\theta_{j}$ 's, the primes $M_{j} / M_{1}$ lie over distinct primes of $k\left[\bar{\theta}_{1}, \ldots, \bar{\theta}_{t-1}\right]$ for $j=1, \ldots, t$. Thus $\operatorname{dim} k\left[\bar{\theta}_{1}, \ldots, \bar{\theta}_{t-1}\right] \geqslant t-1$ and by (A) the elements $\bar{\theta}_{1}, \ldots, \bar{\theta}_{t-1}$ are algebraically independent over $k$.

Let $I$ denote the kernel of the homomorphism

$$
R\left[X_{1}, \ldots, X_{t-1}\right] \rightarrow R\left[\theta_{1}, \ldots, \theta_{t-1}\right]
$$

given by $X_{j} \rightarrow \theta_{j}$. If we follow $\tau$ by $\sigma$ we get a mapping

$$
R\left[X_{1}, \ldots, X_{t-1}\right] \stackrel{\text { oㄷ }}{\longrightarrow}(R / q)\left[\bar{\theta}_{1}, \ldots, \bar{\theta}_{t-1}\right] \cong(R / q)\left[X_{1}, \ldots, X_{t-1}\right]
$$

which clearly has kernel $q\left[X_{1}, \ldots, X_{t-1}\right]$. Thus $I \subset q\left[X_{1}, \ldots, X_{t-1}\right]$. Moreover, since $I \cap R=0$, the containment is strict. Again using the fact that 
$I \cap R=0$ we localize the exact sequence

$$
0 \rightarrow I \rightarrow R\left[X_{1}, \ldots, X_{t-1}\right] \stackrel{\tau}{\rightarrow} R\left[\theta_{1}, \ldots, \theta_{t-1}\right] \rightarrow 0
$$

at the multiplicative system $R \backslash 0$. This yields an exact sequence

$$
0 \rightarrow I\left(K\left[X_{1}, \ldots, X_{t-1}\right]\right) \rightarrow K\left[X_{1}, \ldots, X_{t-1}\right] \rightarrow K \rightarrow 0 .
$$

Thus $I \cdot\left(K\left[X_{1}, \ldots, X_{t-1}\right]\right)$ has rank $t-1$ and it follows that $I$ has rank $t-1$. Recalling that $t-1 \leqslant n$, we have now shown that $P_{t}=q\left[X_{1}, \ldots, X_{n}\right]$ contains the prime $I\left[X_{t}, \ldots, X_{n}\right]$ which is the kernel of the homomorphism

$$
R\left[X_{1}, \ldots, X_{n}\right] \rightarrow R\left[\theta_{1}, \ldots, \theta_{t-1}\right]\left[X_{t}, \ldots, X_{n}\right]
$$

which takes $X_{i}$ to $\theta_{i}$ if $1 \leqslant i \leqslant t-1$ and $X_{j}$ to $X_{j}$ if $j \geqslant t$. Since

$$
t-1=\operatorname{rank} I \leqslant \operatorname{rank} I\left[X_{t}, \ldots, X_{n}\right]<\operatorname{rank} q\left[X_{1}, \ldots, X_{n}\right]=t,
$$

we see that rank $I\left[X_{t}, \ldots, X_{n}\right]=t-1$. We can now modify our original chain $0<P_{1}<\cdots<P_{t}<\cdots<P_{n}$ so that $P_{t-1}=I\left[X_{t}, \ldots, X_{n}\right]$. Hence we have the computation

$$
\begin{aligned}
{[s-(t-1)] } & =\operatorname{dim} R\left[X_{1}, \ldots, X_{n}\right] / P_{t-1}=\operatorname{dim} R\left[X_{1}, \ldots, X_{n}\right] / I\left[X_{t}, \ldots, X_{n}\right] \\
& =\operatorname{dim} R\left[\theta_{1}, \ldots, \theta_{t-1}\right]\left[X_{t}, \ldots, X_{n}\right] .
\end{aligned}
$$

There are now two possibilities, each of which leads to a contradiction.

Case 1. $t>1$. In this case, by the minimality of $n$ there exist $\gamma_{t}, \ldots, \gamma_{n}$ in $K$ such that

$$
\begin{aligned}
s-(t-1) & =\operatorname{dim} R\left[\theta_{1}, \ldots, \theta_{t-1}, X_{t}, \ldots, X_{n}\right] \\
& =[n-(t-1)]+\operatorname{dim} R\left[\theta_{1}, \ldots, \theta_{t-1}, \gamma_{t}, \ldots, \gamma_{n}\right] .
\end{aligned}
$$

That is,

$$
\operatorname{dim} R\left[\theta_{1}, \ldots, \theta_{t-1}, \gamma_{t}, \ldots, \gamma_{n}\right]+n=s=\operatorname{dim} R\left[X_{1}, \ldots, X_{n}\right] .
$$

This is a contradiction.

Case 2. $t=1$. In this case $P_{1}=q\left[X_{1}, \ldots, X_{n}\right]$ and we have

$$
s-1=R\left[X_{1}, \ldots, X_{n}\right] / q\left[X_{1}, \ldots, X_{n}\right]=R / q\left[X_{1}, \ldots, X_{n}\right] .
$$

By the minimality of $\operatorname{dim} R$, there are $\bar{\theta}_{1}, \ldots, \bar{\theta}_{n}$ in the quotient field of $R / q$ such that

$$
\operatorname{dim}(R / q)\left[X_{1}, \ldots, X_{n}\right]=\operatorname{dim}(R / q)\left[\bar{\theta}_{1}, \ldots, \bar{\theta}_{n}\right]+n .
$$

Let $\lambda: R \rightarrow R / q$ be the canonical homomorphism. Then $\lambda$ extends to a mapping of $R_{q}$ onto the quotient field of $R / q$. Choose $\theta_{1}, \ldots, \theta_{n}$ in $R_{q}$ such that $\lambda\left(\theta_{i}\right)=\bar{\theta}_{i}$. Then we have an induced homomorphism

$$
R\left[\theta_{1}, \ldots, \theta_{n}\right] \stackrel{\lambda}{\longrightarrow}(R / q)\left[\theta_{1}, \ldots, \theta_{n}\right] .
$$

Since this homomorphism has a nontrivial kernel, 


$$
\begin{aligned}
\operatorname{dim} R\left[\theta_{1}, \ldots, \theta_{n}\right] & \geqslant \operatorname{dim}(R / q)\left[\bar{\theta}_{1}, \ldots, \bar{\theta}_{n}\right]+1 \\
& =\operatorname{dim}(R / q)\left[X_{1}, \ldots, X_{n}\right]-n+1=s-1-n+1=s-n .
\end{aligned}
$$

Thus

$$
\operatorname{dim} R\left[\theta_{1}, \ldots, \theta_{n}\right]+n \geqslant \operatorname{dim} R\left[X_{1}, \ldots, X_{n}\right] .
$$

Thus to complete our argument we need only show that $\operatorname{dim} R\left[\theta_{1}, \ldots, \theta_{n}\right]$ $+n \leqslant \operatorname{dim} R\left[X_{1}, \ldots, X_{n}\right]$. But, as mentioned earlier, this is true for any set of $\theta$ 's. For consider the exact sequence

$$
0 \rightarrow J \rightarrow R\left[X_{1}, \ldots, X_{n}\right] \rightarrow R\left[\theta_{1}, \ldots, \theta_{n}\right] \rightarrow 0
$$

given by the homomorphism $X_{i} \rightarrow \theta_{i}$. We surely have $\operatorname{dim} R\left[X_{1}, \ldots, X_{n}\right]$ $\geqslant \operatorname{dim} R\left[\theta_{1}, \ldots, \theta_{n}\right]+\operatorname{rank} J$. We need only show that $\operatorname{rank} J=n$. Since $J \cap R=0$, we may localize at the multiplicative system $R \backslash 0=S$ and compute the rank of $J_{s}$. After localizing we have

$$
0 \rightarrow J_{s} \rightarrow K\left[X_{1}, \ldots, X_{n}\right] \rightarrow K \rightarrow 0 .
$$

If we now apply (A) we compute rank $J_{s}=n$.

\section{REFERENCES}

1. J. T. Arnold, On the dimension theory of overrings of an integral domain, Trans. Amer. Math. Soc. 138 (1969), 313-326. MR 39 \# 188.

2. J. Arnold and R. Gilmer, On the dimension sequence of a commutative ring, Amer. J. Math. 90 (1974), 385-408.

3. N. Bourbaki, Eléments de mathématique. Algèbre commutative, Actualités Sci. Indust., nos. 1290, 1293, 1308, Hermann, Paris, 1961-1965. MR 30 \#2027; 33 \#2660; 36 \# 146; 41 \# 5339.

4. E. Bastida and R. Gilmer, Overrings and divisorial ideals of rings of the form $D+M$, Michigan Math. J. 20 (1973), 79-95. MR 48 \#2138.

5. J. Brewer, P. Montgomery, E. Rutter and W. Heinzer, Krull dimension of polynomial rings, Conference on Commutative Algebra, Lecture Notes in Math., vol. 311, Springer-Verlag, New York, 1973.

6. P. Jaffard, Théorie de la dimension dans les anneaux de polynomes, Mémor. Sci. Math., fasc. 146, Gauthier-Villars, Paris, 1960. MR 22 \#8038.

7. W. Krull, Jacobsonsche Ringe, Hilbertscher Nullstellensatz, Dimensions theorie, Math. Z. 54 (1951), 354 -387. MR 13, 903.

8. M. Nagata, Local rings, Interscience Tracts in Pure and Appl. Math., no. 13, Interscience, New York and London, 1962. MR 27 \#790.

9. A. Seidenberg, $A$ note on the dimension theory of rings, Pacific J. Math. 3 (1953), 505-512. MR 14, \#941.

10. - On the dimension theory of rings. II, Pacific J. Math. 4 (1954), 603-614. MR 16, 441.

Department of Mathematics, University of Kentucky, Lexington, Kentucky 40506 